\section{A) Check for updates}

Cite this: Food Funct., 2021, 12, 9844

\title{
Fructooligosaccharide supplementation alleviated the pathological immune response and prevented the impairment of intestinal barrier in DSS- induced acute colitis mice $\uparrow$
}

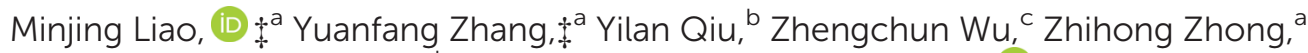 \\ Xiaoqi Zeng, ${ }^{a}$ Yiliang Zeng, ${ }^{d}$ Li Xiong, ${ }^{*}{ }^{\mathrm{e}}$ Yu Wen ${ }^{*}$ and Rushi Liu (D)*a
}

\begin{abstract}
The dysbiosis of gut microbiota is closely related to the occurrence and development of inflammatory bowel disease (IBD). The manipulation of intestinal flora through prebiotics or probiotics is expected to induce and maintain the remission of IBD symptoms. 6-week-old C57BL/J mice were daily gavaged with fructooligosaccharides (FOS) or the synbiotic two weeks before the administration of dextran sulfate sodium (DSS). The supplementation of FOS or synbiotic could significantly ameliorate the body weight loss and colon histological damage in DSS-induced acute colitis mice. The altered composition of gut microbiota in acute colitis mice was reversed by FOS or Synbiotic supplementation, with a characteristic of decreased abundance of Mucispirillum. Both FOS and synbiotic mitigated DSS-induced loss of mucus protein (MUC2) and epithelium tight junction proteins (ZO-1, Occluding, Claudin1) in colon mucosa. The expression of pro-inflammatory cytokines (IL- 6 and TNF- $\alpha$ ) was decreased by FOS or synbiotic treatment, while the expression of Tbx21 and IL-10 was increased. The results suggested that the modulation of gut microbiota by FOS or synbiotic supplementation could decrease the inflammation potential of colonized commensals, which prevented the impairment of the intestinal barrier and induced a regulation of immune response in DSS-induced acute colitis mice.
\end{abstract}

Received 14th April 2021 Accepted 24th August 2021

DOI: 10.1039/d1fo01147b

rsc.li/food-function

\section{Introduction}

Inflammatory bowel disease (IBD) is a kind of chronic idiopathic intestinal inflammatory disease, mainly including ulcerative colitis and Crohn's disease. With the westernization of lifestyle, the incidence of IBD has increasingly raised in newly industrialized countries, such as China. ${ }^{1,2}$ It is characterized by early age of onset, frequent recurrence, and high risk of cancer. The routine strategy for IBD treatment mainly includes use of anti-inflammatory drugs, corticosteroids, immunosuppressive agents, and a compound of tumor necrosis factor (TNF) monoclonal antibodies to suppress intestinal inflammation. Although current therapies can ameliorate

\footnotetext{
${ }^{a}$ Department of Medical Laboratory, School of Medicine, Hunan Normal University, Changsha 410013, China. E-mail: liurushi@hunnu.edu.cn

${ }^{b}$ School of Life Science, Hunan Normal University, Changsha 410018, China ${ }^{c}$ Hunan Cancer Hospital, Changsha 410013, China

${ }^{d}$ Shaoshan Changbaitong Biotechnology Co., Ltd., Shaoshan 411100, China ${ }^{e}$ General Surgery Department, Second Xiangya Hospital, Central South University, Changsha 410011, China. E-mail: lixionghn@csu.edu.cn,wenyu2861@csu.edu.cn $\dagger$ Electronic supplementary information (ESI) available. See DOI: 10.1039/ d1fo01147b

$\$$ These authors make equal contribution to this paper.
}

symptoms in the active phase of IBD, still some patients do not respond to these treatments or cannot tolerate them due to their significant side effects, and most patients may experience frequent recurrence because the disease is still incurable. $^{3}$

The interaction of gut microbiota, epithelial cells, and immune system was considered to play an important role in the pathogenesis of IBD. ${ }^{4}$ Although it is not clear whether the increase of potential pathogenic bacteria or the loss of protective strains has a bigger impact on IBD susceptibility and morbidity, the disease progression and gastrointestinal symptoms are closely related to the composition and function of intestinal flora. ${ }^{5,6}$ The widespread clinical experience and many preclinical studies suggested that modulation of gut microbiota through administration of beneficial bacterial species (probiotics), poorly absorbed dietary oligosaccharides (prebiotics), or combined prebiotics and probiotics (synbiotic) was a very promising option for treating IBD in combination with traditional anti-inflammatory and immunosuppressive agents. ${ }^{7,8}$

In the past decades, many studies focused on the effects of probiotics in the prevention or treatment of IBD, especially the Lactobacillus and Bifidobacterium strains. ${ }^{9-11}$ Bifidobacterium lactis was found to inhibit NF-kB in intestinal epithelial cells. ${ }^{12}$ 
Lactobacillus reuteri was found to maintain a functional mucosal barrier by decreasing the bacterial translocation during DSS treatment. $^{13}$ Lactobacillus fermentum and Bifidobacterium animal could aid in the regeneration of intestinal epithelium in DSS-treated mice. ${ }^{14}$ Current evidence indicated that the intervention effects of probiotics are strain specific, and their efficacy were more certain in the treatment of patients with ulcerative colitis or chemically induced colitis animal models.

Compared with probiotics, prebiotics are nontoxic and easy to store, can modulate a wide range of gut commensals, and won't transfer bacterial resistance gene. Many kinds of prebiotics were proved to favor the growth of bifidobacteria or the production of short-chain fatty acids such as butyrate, indicating their potential benefits to treat inflammatory bowel diseases. ${ }^{15}$ Fructooligosaccharides (FOS) is a widely used prebiotic, which might exert anti-inflammatory effects through immunomodulation or indirectly through the microbiota. ${ }^{16} \mathrm{~A}$ few studies had suggested the beneficial effects of FOS to suppress gut inflammation in chemically induced rodent models of colitis, ${ }^{17-19}$ while studies on the intervention effects of FOS in patients with Crohn's disease showed contradictory results. $^{20,21}$

Recently, several studies suggested that the use of probiotic Lactobacillus or Bifidobacterium with prebiotic as a synbiotic combination was more efficacious than either the probiotic or prebiotic alone to alleviate the intestinal inflammation and prevent the dysbiosis of gut microbiota caused by DSS treatment. $^{22-24}$ In present study, we explored the intervention effects and mechanisms of FOS and the synbiotic (FOS combined with several probiotics mainly from Bifibacterium and Lactobacillus) in DSS-induced colitis mice.

\section{Materials and methods}

\subsection{Animals}

The manipulation of animals was approved by Ethics Committee Board of Hunan Normal University, and performed according to the guidelines in accordance with National Institutes of Health guide for the care and use of Laboratory animals (NIH Publications no. 8023, revised 1978). Six-weekold healthy male C57BL/6 mice were purchased from Hunan SJA laboratory animal company and maintained in specificpathogen-free environment under normal conditions (at a temperature of $21-23{ }^{\circ} \mathrm{C}$ and a humidity of $40-60 \%$, on a $12 \mathrm{~h}$ light/dark cycle). The mice were housed in groups of 4-5 per cage with free access to food and water.

\subsection{Prebiotic and synbiotic}

The prebiotic used in present study is fructo-oligosaccharide (FOS). FOS (P95S) was manufactured by Quantum Hi-Tech Biological Company and gifted by Mr Xiaolin Chen. The synbiotic used in present study was composed of FOS and 8 probiotic strains, including Lactobacillus reuteri, Lactobacillus paracasei, Lactobacillus fermentum, Lactobacillus rhamnosus,
Bifidobacterium lactis, Bifidobacterium breve, Bifidobacterium animal and Streptococcus thermophilus.

\subsection{Experimental design}

After a week of adaptation to the laboratory conditions, the mice were assigned randomly to four groups, as Control group, DSS-induced colitis group (DSS), FOS-treated colitis group (DSS_FOS), and synbiotic-treated colitis group (DSS_Synbiotic). Each group included 8-10 mice (19 $\pm 1 \mathrm{~g})$ and were fed with ordinary rodent fodder, while mice in group DSS_FOS and DSS_Synbiotic were daily administered with FOS (2 g per kg b. w.) or the synbiotic (FOS $2 \mathrm{~g}$ per $\mathrm{kg}$ b.w. + probiotic $10^{9} \mathrm{CFU}$ per kg b.w.) by gavage. Two weeks later, the mice were subjected to the procedure of DSS-induced colitis, except for Control group mice.

\subsection{Procedure of DSS-induced colitis}

The mice in control group were free to drink sterilized water every day, while the mice in other groups were subjected to drink dextran sulfate sodium (DSS) solution to induce colitis. Mice were subjected to drink 3\% DSS solution daily for 7 days to induce acute colitis. Mice that had underwent 3 rounds of DSS administration were considered to have chronic colitis. Each round included 7 days of $2 \%$ DSS solution drinking, and 7 days of sterilized water drinking.

\subsection{Mice colonoscopy and sample collection}

The colorectal mucosa was observed directly under the mouse live enteroscope. The anesthetized mice were fixed in supine position on a clean operating table, and their enteric cavity were rinsed with normal saline through anus to remove feces. Insert the endoscope (Guangzhou Red Pine Medical Instrument Co., Ltd, China) through the anus, and gently reach the splenic flexure of colon. Inject the gas intermittently to ensure the intestinal cavity is filling to get a clear display of intestinal mucosa. Then draw back the endoscope slowly and record the images by software. 2 days after the completion of DSS modeling, all mice were anesthetized by intraperitoneal injection of pentobarbital sodium, then sacrificed by decapitation for sampling. The intestine of each mouse was quickly isolated, and the colon length was measured. $1 \mathrm{~cm}$ of distal colon segment was taken for tissue section and HE staining, and the pathological damage of colon tissue of mice in each group were evaluated. $2 \mathrm{~cm}$ of colonic segments adjacent to the caecum were collected to analyze the gene expression and gut microbiota.

\subsection{Histological analysis}

The intestinal segments were cut longitudinally, rinsed with phosphate buffer, and fixed in $10 \%$ neutral formalin for 24 hours. Then the specimens were prepared to be embedded in paraffin for tissue section. Each group included 5 mice at least, and 3 randomly selected sections per mouse were used to evaluate the colon impairment. $3 \mu \mathrm{m}$-thick sections were deparaffinized with xylene and rehydrated through graded alcohols, and then counterstained with hematoxylin and eosin. 
The slides were finally dehydrated with grade alcohols, soaked in xylene, and mounted with neutral balsam. The images were captured by a light microscope (Olympus CX41), and assessed by two independent researchers. The pathological score was assessed according to the degree of tissue damage and inflammatory cell infiltration, based on the criteria described by Wirtz et al. ${ }^{25}$

\subsection{Amplicon sequencing and bioinformatics analysis}

Obtained colon samples were delivered to the laboratory in an icebox and stored at $-80{ }^{\circ} \mathrm{C}$. Total genomic DNA was extracted using QIAGEN DNA Extraction kit following the manufacturer's instructions. Extracted DNA was diluted to a concentration of $1 \mathrm{ng} \mathrm{\mu l}^{-1}$ and used as template for PCR amplification of bacterial 16s rDNA with barcoded primers and Takara Ex Taq (Takara) for amplicon sequencing. For bacterial diversity analysis, V3-V4 variable regions of 16s rDNA was amplified with universal primers $343 \mathrm{~F}$ and 798R. After 2 rounds of PCR, the final amplicon was purified with the AMPure XP beads (Agencourt) and quantified using Qubit dsDNA assay kit. Equal amounts of purified amplicon were pooled for subsequent sequencing on Illumina MiSeq, conducted by $\mathrm{OE}$ Biotech Company (Shanghai, China). The raw sequencing data were filtered to eliminate the adapter pollution and low quality to obtain clean reads by Trimmomatic software (version 0.35), and then paired-end reads were merged to tags by FLASH software (version 1.2.11). Clean tags were clustered into Operational Taxonomic Units (OTU) at 97\% sequence similarity by Vsearch software (version 2.4.2). All representative reads were annotated and blasted against Greengens database using Ribosomal Database Project Classifier (0.60 confidence values as cutoff). Abundance differences of microbial communities between samples were assessed with the statistical methods based on OTU and taxonomic ranks.

\subsection{Transcriptome sequencing and bioinformatics analysis}

Total RNA was extracted from colon tissues using the RNA Isolation KIT (Ambion, AM1561) following the manufacturer's protocol, and the samples with RNA integrity number $\geq 8$ were subjected to the subsequent analysis. The libraries were constructed using TruSeq Stranded mRNA LTSample Prep Kit (Illumina, CA, USA). Then these libraries were sequenced on the Illumina sequencing platform (HiSeq X-ten) and $125 \mathrm{bp} /$ $150 \mathrm{bp}$ paired-end reads were generated. The sequencing and transcriptome analysis were conducted by OE Biotech Company (Shanghai, China). The clean reads were mapped to reference genome using hisat2 software. FPKM (Fragments Per $\mathrm{kb}$ Per Million Reads) value of each gene was calculated using cufflinks software and the read counts of each gene were obtained by htseq-count software. Differentially expressed genes (DEGs) were identified using DESeq. $P$ value $<0.05$ and foldChange $>2$ or foldChange $<0.5$ was set as the threshold for significantly differential expression. GO enrichment and KEGG pathway enrichment analysis of DEGs were respectively performed based on the hypergeometric distribution.
Hierarchical cluster analysis of DEGs was performed using the OECloud tools at https://cloud.oebiotech.cn.

\subsection{Real-time quantitative PCR (qPCR)}

The colon tissue samples prepared for qPCR detection were homogenized with AG RNAex Pro reagent (AG21102) for total RNA extraction. Then total RNAs were reverse-transcripted to cDNA using Evo M-MLV RT Kit (AG11705). qPCR was performed with SYBR Premix Pro Taq HS qPCR kit (AG11701) on CFX Connect system (Bio-Rad Co. Ltd, United States). The reactions were all carried out with a two-step program: 30 seconds at $95{ }^{\circ} \mathrm{C}$ followed by 40 cycles of 5 seconds at $95{ }^{\circ} \mathrm{C}$ and 30 seconds at $60{ }^{\circ} \mathrm{C}$. The reagents for RNA extraction and RT-PCR were all purchased from Hunan Accurate Biotech. Co. Ltd, China. The primers for reference and target genes were synthesized from Tsingke Biological Technology Company (Changsha, Hunan, China), and primer sequences were provided in ESI. $\dagger$

\subsection{Western blotting (WB)}

The colon tissue samples prepared for WB detection were homogenized with RIPA lysis buffer (CW2334S, CWBIO Co. Ltd, China) for protein extraction. Proteins were separated by sodium dodecyl sulfate poly-acrylamide gel electrophoresis and electrophoretically transferred to polyvinylidene fluoride membrane. After blocking, the membranes were incubated with primary antibody (diluted as 1 : 1000) against at $4{ }^{\circ} \mathrm{C}$ overnight. After washing, the membranes were further incubated with HRP-conjugated second antibody at a dilution of $1: 5000$ (goat anti-rabbit IgG, AP132P, Millipore Co. Ltd) (goat antimouse IgG, abs20001, Absin Bioscience Co. Ltd, China). The immune complexes were detected with ECL reagent (130231, Monad Biotech. Co. Ltd, China), and the chemiluminescence signal was imaged by Tanon 5500 system (Tanon Co. Ltd, Shanghai, China). The quantitation analysis was performed using ImageJ software and normalized to $\beta$-actin band intensity on the same membrane. The primary antibodies against $\beta$-actin, Claudin1, Occludin, ZO-1, MUC2 and TNF- $\alpha$ were all purchased from Proteintech Group Inc., China.

\subsection{Immunohistochemistry (IHC) analysis}

The expression of MUC2 and tight junction proteins (Claudin1, Occludin, ZO-1) were analyzed by immunohistochemistry assay as follows. The antibodies used for IHC were same as that for WB. $3 \mu \mathrm{m}$-thick sections were de-paraffinized with xylene and rehydrated through graded alcohols, and then treated with $0.01 \mathrm{M}$ sodium citrate for antigen retrieval. The sections were incubated with $3 \% \mathrm{H}_{2} \mathrm{O}_{2}$ in the dark for 12 minutes to quench endogenous peroxidase activity, and then incubated with goat serum at room temperature for 30 minutes for blocking. The sections were incubated with primary antibody (diluted as $1: 500$ ) at $4{ }^{\circ} \mathrm{C}$ overnight. Then the slides were rinsed with PBST 4 times, and incubated with respective HRP-conjugated secondary antibody (diluted as $1: 1000)$ at $37^{\circ} \mathrm{C}$ for 45 minutes. The slides were rinsed with PBST 4 times, and then incubated with DAB (ZLI-9017, ZSGB 
Biological Co. Ltd, Beijing, China) for 1 minute and immediately washed under tap water after color development. Slides were counter stained with hematoxylin for 90 seconds. The sections were finally dehydrated with grade alcohols, soaked in xylene, and mounted with neutral balsam. The images were captured by a light microscope (Olympus CX41), and the optical density were analyzed by IPP 6.0 software.

\subsection{Statistical analysis}

The statistical analyses were carried out by GraphPad Prism 7 (GraphPad Software, USA). Data were analyzed for normal distribution before any statistical analyses. The values were expressed as means \pm standard deviation (SD), except for the individual values of bacterial relative abundance which were presented as scatter plots with column bar graphs or as box plots showing the interquartile range and median value. The statistical significance between two groups was determined with two-way unpaired Student's $t$ test, Mann-Whitney test, or Wilcoxon Rank-Sum test. The significance in difference was assigned at the level of $<5 \%$ probability $(P<0.05)$.

\section{Results and discussion}

\subsection{FOS supplementation ameliorated DSS-induced acute colitis in mice}

It was generally thought that the alleviation of colitis required the prebiotic given prior to DSS treatment. ${ }^{26}$ Therefore, the mice were supplemented with FOS or synbiotic two weeks before the DSS administration. Based on our experience in previous study, ${ }^{27}$ we administrated FOS at a daily dose of $2 \mathrm{~g}$ per $\mathrm{kg}$ b.w. in present study, which was about equivalent to the diet supplemented with 1\% FOS. This dose was well-tolerated for weeks of administration in adult inbred mice and didn't exert significant effects on health condition.

a
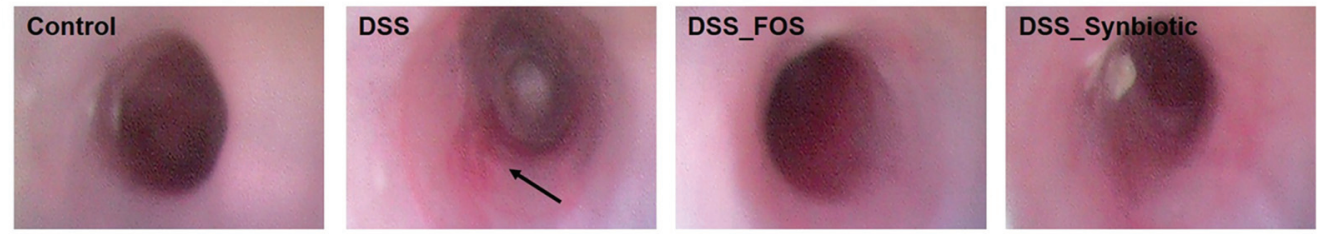

b
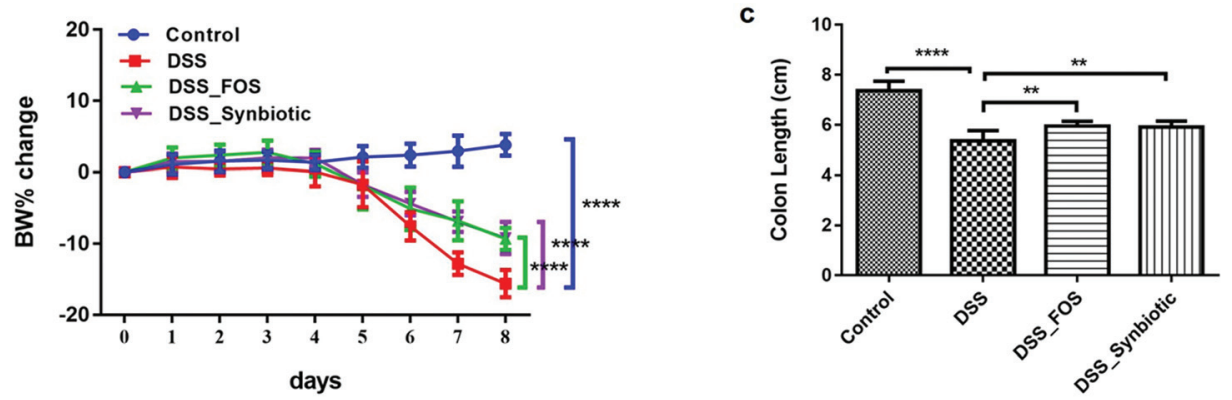

d
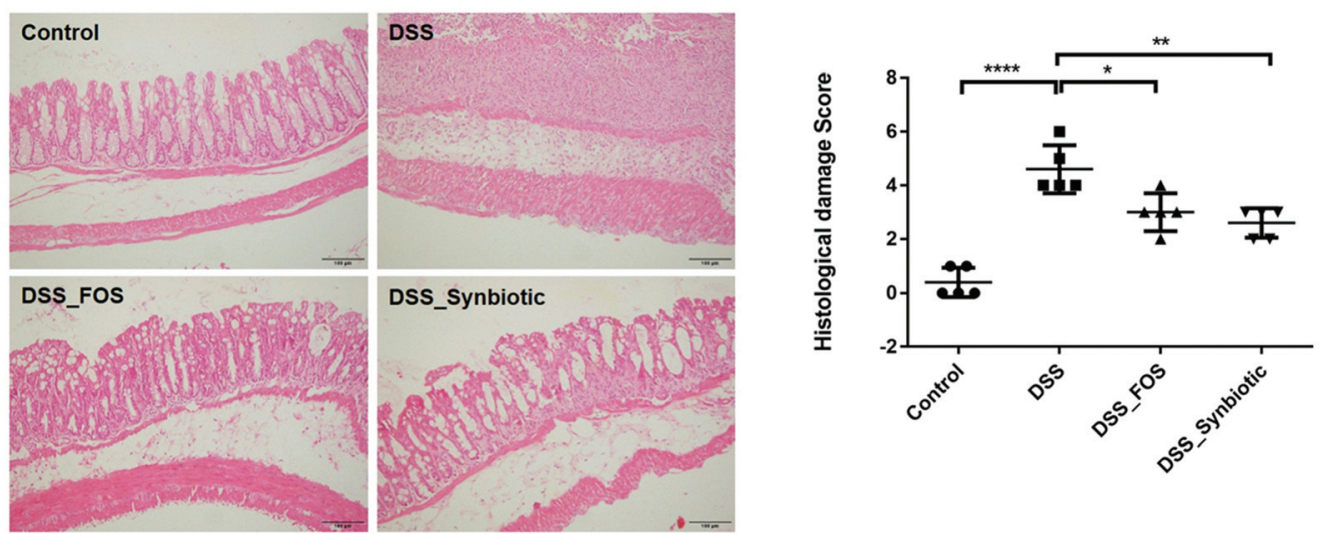

Fig. 1 FOS supplementation attenuated disease activity in acute colitis mice. (a) Colonoscopy examination in each group mice. The arrow indicated the ulcer on colon mucosa. The colitis activity was evaluated through measurement of body weight change (b), colon length (c) and histological damage score (d). $P$ values were analyzed by two-tailed unpaired $t$-test (b and $\mathrm{c}$ ) or two-tailed unpaired Mann-Whitney test (d). * $P<0.05$, $* * P<$ $0.01,{ }^{* * *} P<0.0001, n \geq 5$. 
DSS-induced colitis in mice share many similarities with ulcerative colitis in human, characteristically with a significant decrease in body weight, bloody diarrhea, multiple ulcers, loss of intestinal epithelium cells and infiltration of inflammatory cells. $^{25}$ As illustrated in Fig. 1, a week of DSS administration successfully induced acute colitis in $\mathrm{C} 57 \mathrm{BL} / 6$ mice, while the supplementation of FOS and synbiotic significantly prevented the body weight loss and colon damage in acute colitis mice. The improvement of disease activity index or histological damage score in acute colitis mice presented no significant difference between FOS and synbiotic treatment.

We also examined the intervention effects of FOS and synbiotic in DSS-induced chronic colitis mice. As illustrated in ESI Fig. $1, \dagger$ compared with untreated chronic colitis mice, FOStreated mice presented less ulcers and obvious mucus accumulation on colon mucosa, and the DSS-induced alterations in colon length and histological damage score were attenuated in FOS-treated mice, suggesting that FOS could also partly a

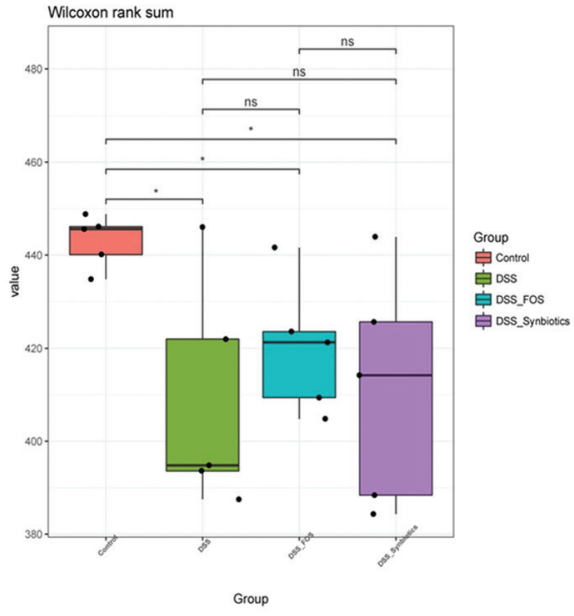

C

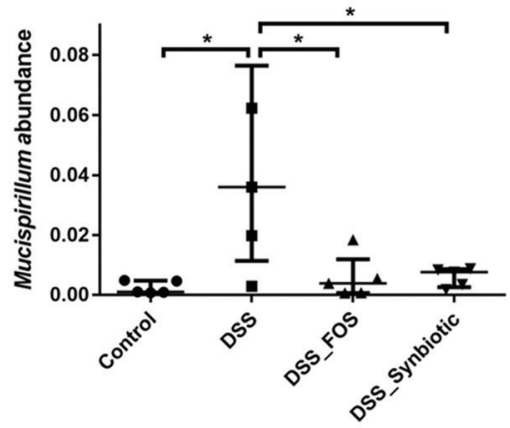

b

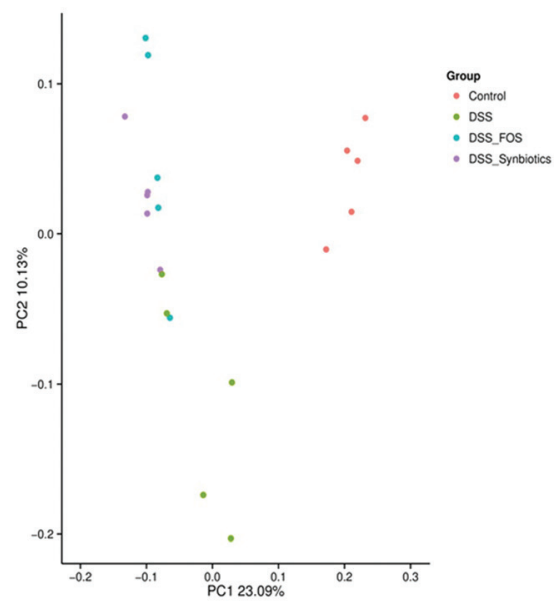

d

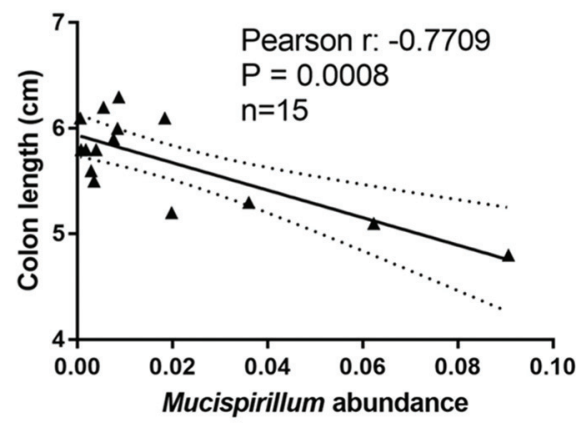

e

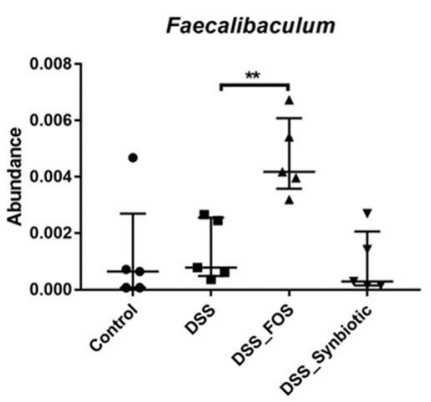

f

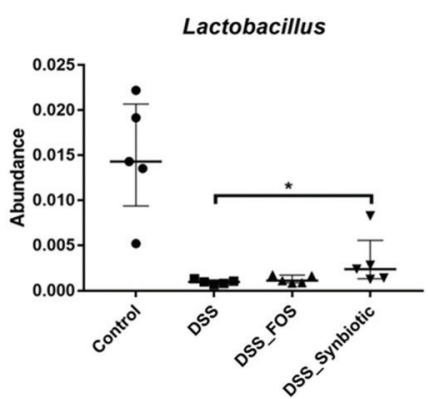

g

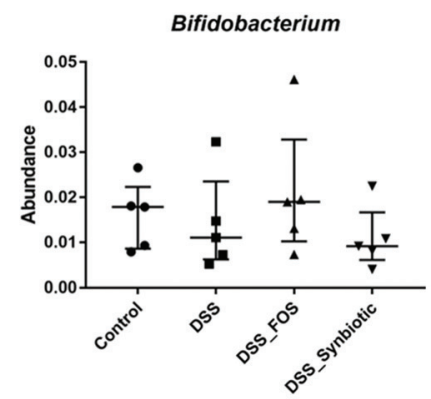

Fig. 2 FOS supplementation modulated the gut microbiota in acute colitis mice. The microbial composition of colon mucosa was analyzed by $16 \mathrm{~s}$ rDNA sequencing in each group mice. The alpha and beta diversity of microbial composition among groups were presented by the Observed species boxplot (a) and the Principal coordinate analysis (b). Abundance of Mucispirillum among groups (c). Correlation analysis between the abundance of Mucispirillum and colon length (d). Relative abundance of Faecalibaculum (e), Lactobacillus (f) and Bifidobacterium (g) among groups. The pairwise comparisons were performed by Wilcoxon rank sum test (c and $\mathrm{e}-\mathrm{g}$ ). ${ }^{*} P<0.05,{ }^{*} P<0.01, n=5$. 
prevent the colon damage in chronic colitis mice. Inflammatory bowel diseases are chronic inflammatory disorders. Although the usefulness of FOS needs to be further assessed in different animal models of colitis and clinical studies, the protective effects of FOS in DSS-induced acute and chronic colitis mice indicated it as a promising dietary supplement or functional food to treat IBD.

As illustrated in Fig. 1 and ESI Fig. $1, \uparrow$ the synbiotic only significantly attenuated the colon damage in DSS-induced acute colitis mice. Compared with untreated mice, the synbiotic-treated chronic colitis mice had an increased tendency of body weight loss. A previous study also found that the treatment of two probiotic strains (Lactobacillus rhamnosus and Bifidobacterium breve) exerted beneficial effects only in active phase of DSS-induced colitis. ${ }^{28}$ Clinical studies suggested Escherichia coli Nissle 1917 and VSL\#3 shown strongest efficacy in the maintenance of remission in patients with ulcerative colitis. $^{29,30}$ In present study, we also didn't find any synergistic effect between FOS and probiotic mixture. The use of synbiotic in inflammatory bowel diseases should take many factors such as disease course, probiotic strain, and dosage into consideration. $^{31}$

\subsection{The modulation of gut microbiota by FOS supplementation in acute colitis mice}

Bacteria that ferment fibers and produce short chain fatty acids (SCFAs) are typically reduced in mucosa and feces of patient with IBD. The dysbiosis of gut microbiota has been considered to innitiate and aggravate gut inflammation in the course of IBD. ${ }^{5,8}$ The microbial composition in colon mucosa was analyzed by $16 \mathrm{~S}$ rDNA sequencing after a week of DSS administration. As illustrated in Fig. $2 \mathrm{a}$ and b, compared with the control group, the diversity of gut microbiota in acute colitis mice was significantly decreased, and the microbial composition was significantly altered. DSS-induced decreased diversity of gut microbiota was partly attenuated by FOS or synbiotic supplementation in acute colitis mice (Fig. 2a). A previous study found that 28 days of chow diet supplemented with $10 \%$ FOS caused a decreased diversity of gut microbiota and aggravated the inflammation in ileocecal resection mice. ${ }^{32}$ Therefore, it was generally thought that the modulation of gut microbiota through prebiotics or probiotics should avoid exacerbating the loss of bacterial diversity in IBD patients.

At the phulum level, the abundance of Deferribacteres was significantly increased in untreated acute colitis mice (ESI Fig. 2a and $\mathrm{b} \dagger$ ). At the genus level, the abundance of Allobaculum, Bacteroides, Intestinomonas, Mucispirillum and Oscillibacter was significantly increased, while the abundance of Alistipes and Lactobacillus was significantly decreased (ESI Fig. 2c $\dagger$ ). DSS-induced increased abundance of Mucispirillum was significantly decreased by FOS or synbiotic supplementation (Fig. 2c), and its abundance was correlated to the index of disease activity (such as colon length) in acute colitis mice (Fig. 2d). Mucispirillum has been found to have specialized systems to handle oxidative stress during inflammation, ${ }^{33}$ and recognized as a bacterial indicator in DSS-induced colitis. ${ }^{34}$ In addition, we found the alleviation of gut inflammation by FOS or synbiotic treatment was accompanied with decreased abundance of Mucispirillum.

The abundance of Faecalibaculum was significantly increased in FOS-treated acute colitis mice (Fig. 2e), while the abundance of Lacotbacillus and Bifidobacterium had a tendency a

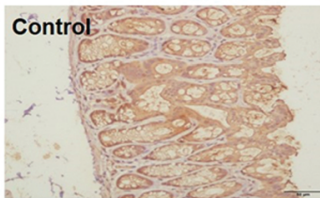

DSS_FOS

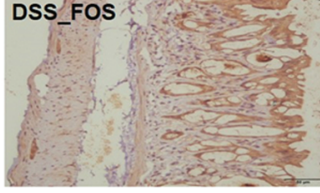

c

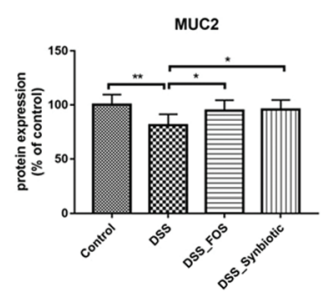

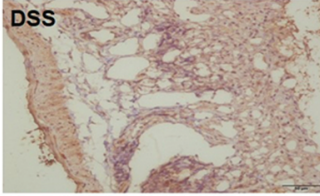

DSS_Synbiotic
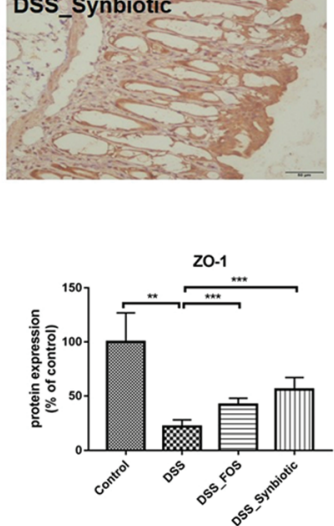

b
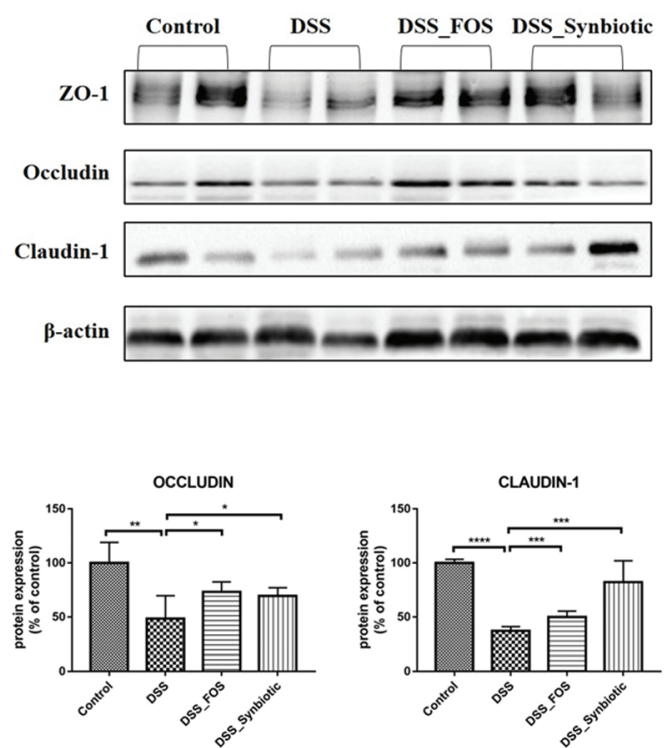

Fig. 3 FOS supplementation prevented the loss of MUC2 and epithelium tight junction proteins in acute colitis mice. The colonic expression of MUC2 in each group mice was examined by immunohistochemistry (a). The colonic expression of ZO-1, Occludin, and Claudin-1 in each group mice were examined by western blot (b). $P$ values were analyzed by two-tailed unpaired $t$-test (c). ${ }^{*} P<0.05,{ }^{* *} P<0.01,{ }^{\star * *} P<0.001, n \geq 5$. 
of increase (Fig. 2f and g). The abundance of Lacotbacillus was significantly increased in synbiotic-treated acute colitis mice (Fig. 2g). Many studies suggested the signatures of micriobial composition and metabolites might be used to predict the action mechanisms of prebiotic or probiotic. ${ }^{24,35-38}$ The presenting results suggested that FOS supplementation might prevent the DSS-induced dysbiosis of gut microbiota, by increasing the abundance of potentially beneficial bacteria (e.g., Faecalibaculum and Bifidobacterium) and decreasing the abundance of potentially harmful bacteria (e.g., Mucispirillum), but the mechanisms need to be further explored.

\subsection{FOS supplementation prevented the impairment of intestinal barrier in acute colitis mice}

The intestinal barrier plays a vital role to protect us from pathogens and commensals-induced inflammation. ${ }^{39}$ The physical structure of intestinal barrier mainly consists of a mucosal layer and a monolayer of epithelial cells. Mucin 2 (MUC2) was the major protein component of mucus, and its decrease was an early event in colitis pathogenesis. ${ }^{40}$ The epithelial cell layer is interconnected by tight junction (TJ) proteins such as Claudins, Occludin and Zonular occludens, which are crucial for the maintenance of epithelial barrier integrity.

As illustrated in Fig. 3 and ESI Fig. $3, \dagger$ the expression of MUC2 and TJ proteins (ZO-1, Occludin, Claudin-1) was significantly decreased after the administration of DSS solution, indicating a destruction of intestinal barrier. The supplementation of FOS or synbiotic could significantly prevented the loss of MUC2 and TJ proteins in DSS-induced acute colitis mice, indicating their protective effects on intestinal barrier. Many studies suggested the supplementation of prebiotic or probiotic could improve the integrity of gut barrier or strengthen the barrier function in animal models of colitis, indicating a therapeutical potential for IBD. ${ }^{41,42}$ a

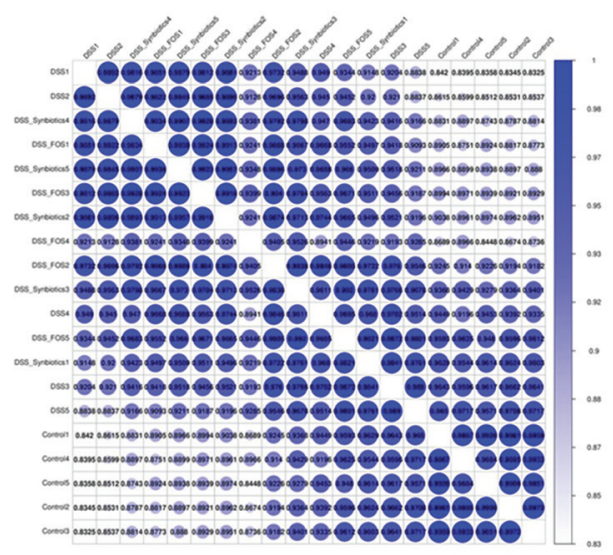

C

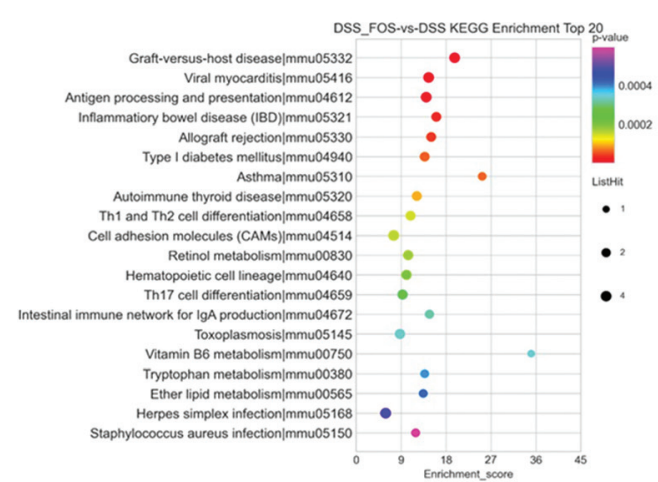

b

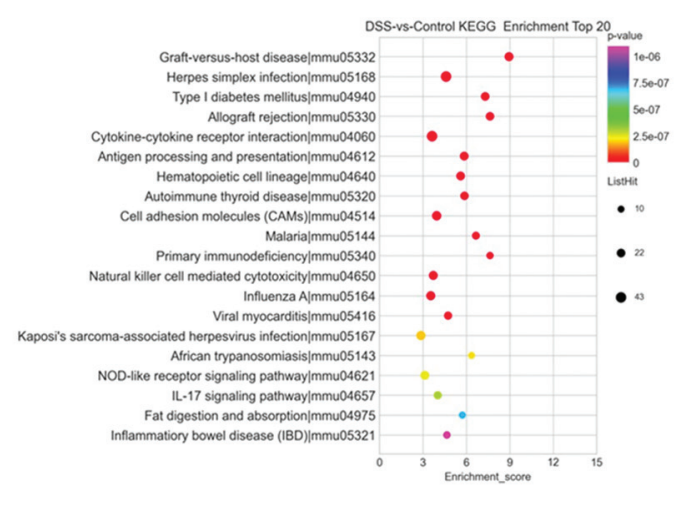

d

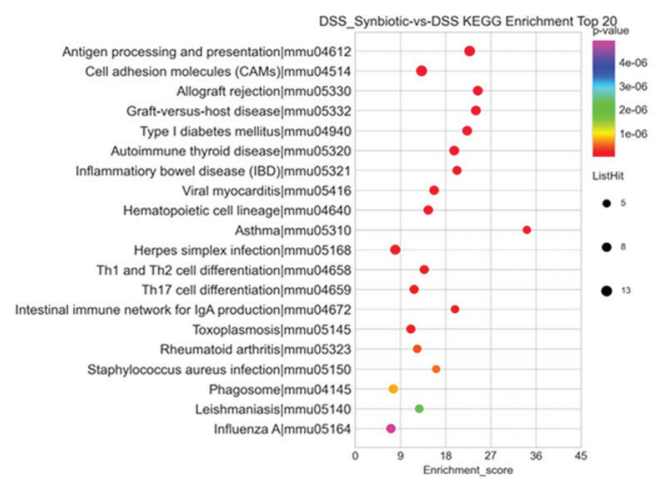

Fig. 4 FOS or synbiotic supplementation partly altered the mRNA expression profile in acute colitis mice. After drinking 3\% DSS solution for a week, the mRNA expression of colon mucosa was analyzed by transcriptome sequencing in each group mice. (a) Heatmap of coefficient matrix among 20 samples. Pearson correlation analysis was performed based on the distribution of FPKM density in each sample, to explore the similarity of expression profile. The darker of the circle color and the bigger of the circle size indicated the stronger of the correlation. (b-d) Bubble diagrams of KEGG enrichment Top 20. Differently expressed genes (DEGs) between two groups underwent pathway analysis using KEGG database. The size of the bubble indicated the number of DEGs, and the color of the bubble indicated the enrichment $p$-value. 


\subsection{FOS supplementation mitigated the pathological immune response in acute colitis mice}

We analyzed the gene expression of colonic mucosa in acute colitis mice through transciptome sequencing. The administration of DSS caused a disastrous disturbance to intestinal environment. As illustrated in Fig. 4a, compared with control mice, the mRNA expression profile was significantly altered in other groups. The use of adjusted $p$-value will be more precise to identify the differentially expressed genes (DEGs). Since the sample size was not large enough in present study, we chose regular $p$-value $<0.05$ as one of criteria to perform DEG analysis. There were 782 DEGs between Control and DSS group, 51 DEGs between DSS and DSS_FOS group, 92 DEGs between DSS and DSS_Synbiotic group (data not shown).

As illustrated in Fig. 4b, the majority of DEGs in DSS group were clustered to pathways of immune system and infectious diseases. Clinical studies had suggested the disordered immune responses driven by gut environment played a vital role in the pathogenesis of IBD. ${ }^{43}$ Although the mechanisms of its colitogenicity are not completely understood, it is widely believed that DSS could cause death of intestinal epithelial cells. The impaired intestinal barrier would allow the gut commensal bacteria to penetrate and reach epithelium, ${ }^{44}$ which might trigger immune responses in gut. As illustrated in Fig. 4c and d, compared with untreated DSS group, the mRNA expression profile occurred slight changes in FOS or synbiotic-treated mice, and most of DEGs were also clustered to pathways of immune system. The results suggested that supplementation of FOS or synbiotic was likely to alleviate the colitis through their immunomodulatory effects, but their effects were insufficient to revert the DSS-induced alterations to gene expression profile.

a

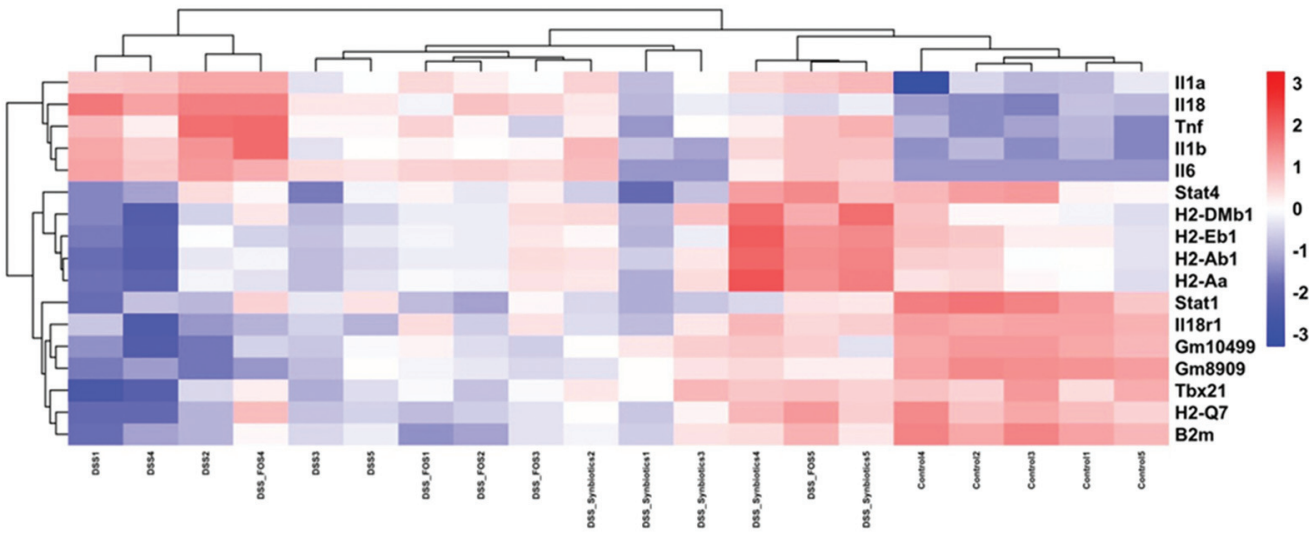

b

c
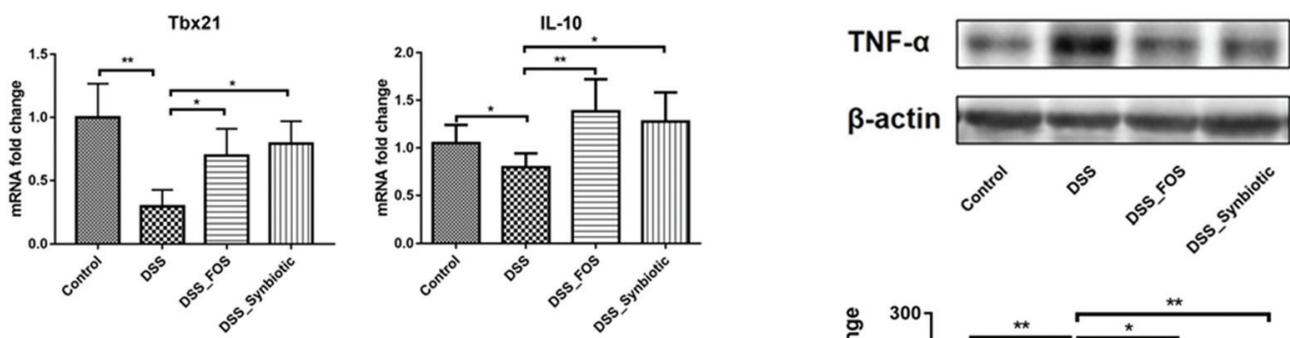

$\beta$-actin
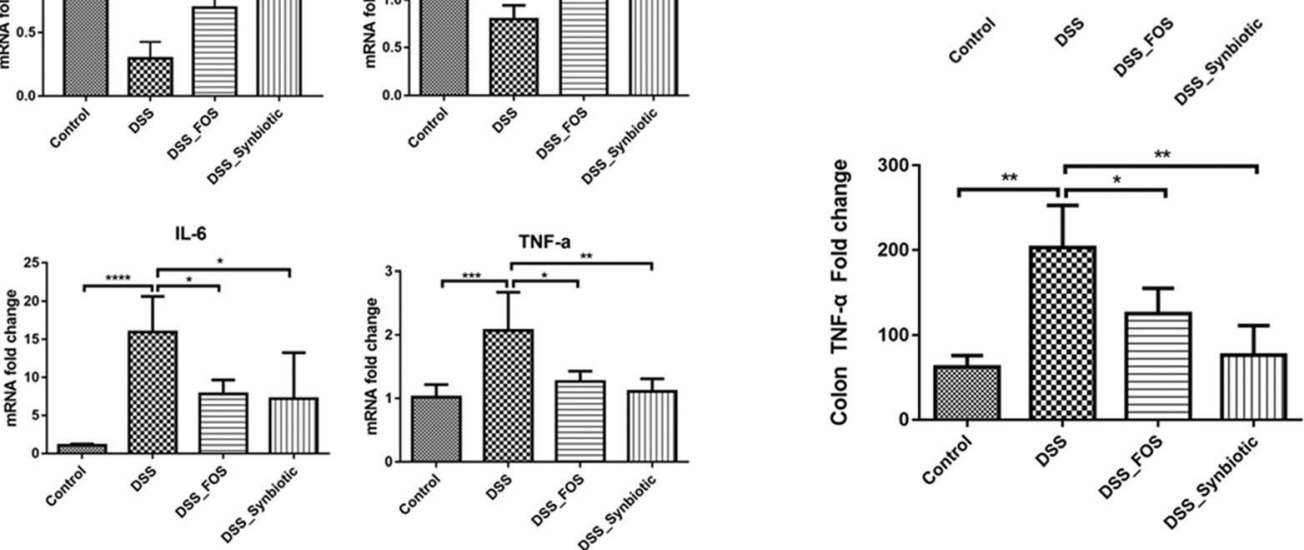

Fig. 5 FOS or synbiotic supplementation regulated the immune response in acute colitis mice. (a) Hierarchical clustering analysis of several differently expressed genes which were related to immune pathways. High expression was shown in red, while low expression was shown in blue. (b) The mRNA expression of Tbx21, IL-6, TNF- $\alpha$ and IL-10 were examined by quantitative PCR. (c) The protein expression of TNF- $\alpha$ were examined by western blot. $P$ values were analyzed by two-tailed unpaired $t$-test ( $d$ and e). ${ }^{*} P<0.05,{ }^{*} P<0.01, n \geq 5$. 
The DSS model of acute colitis is useful to investigate the roles of innate immune mechanisms. We chose several interested DEGs from immune pathways to perform hierarchical clustering analysis. As illustrated in Fig. 5a, the expression of genes which were involved with antigen processing and presentation (such as H2-DMb1 and Gm10499), T helpler cell differentiation (such as Stat1 and Tbx21), was down-regulated in untreated DSS group, while the expression of pro-inflammatory genes (such as Il6 and Tnf) was significantly up-regulated. The supplementation of FOS or synbiotic could partly mitigate above alterations.

Previous studies had found that patients receiving FOS supplementation had reduced proportion of IL-6 positive dentric cells (DCs) and increased proportion of IL-10 positive DCs. ${ }^{20,21}$ TNF- $\alpha$ is a key effector cytokine in IBD and its neutralizing $\mathrm{Ab}$ therapy is widely used in IBD treatment. IL-10 is a vital antiinflammatory cytokine which could suppress the production of pro-inflammatory cytokines such as IL-6 and TNF- $\alpha$. Thus, we further examined the colonic expression of inflammatory cytokines by qPCR and WB (Fig. 5b and c). Compared with untreated acute colitis mice, the expression of IL- 6 and TNF- $\alpha$ was significantly decreased, and the expression of IL-10 was increased in FOS or synbiotic-treated mice. The results suggested that FOS or synbiotic supplementation could dampen the pathogenic immune responses in colonic mucosa of acute colitis mice.

T-BOX21 (Tbx21) or T-box factor expressed in T cells (T-bet) has been found to regulate the production of TNF- $\alpha$ in colon $\mathrm{DCs},{ }^{45}$ and considered to control the innate immune response across mucosal surfaces to avoid pro-inflammatory responses to commensal bacteria. ${ }^{46,47}$ As illustrated in Fig. 5b, we found that FOS or synbiotic supplementation could prevent the DSSinduced decrease of Tbx21 expression in acute colitis mice. The results suggested that FOS might exert immunomodulatory effects by regulating the expression of T-bet in innate immune system, which would be beneficial for the maintenance of intestinal barrier and thus prevented the DSS-induced acute colitis.

\section{Conclusion}

DSS model has been widely used to investigate the effects of gut microbiota and factors causing compositional changes (such as prebiotic) on the development of colitis. In present study, we found the supplementation of FOS or the synbiotic could significantly ameliorate the disease activity in DSSinduced acute colitis mice. The alleviation of colitis activity was correlated with the altered abundance of several gut commensals (such as Mucispirillum and Allobaculum). Both FOS and the synbiotic could significantly prevented DSS-induced loss of Muc2 and epithelium tight junction proteins at colonic mucosa, indicating the protection of intestinal barrier. Meanwhile, FOS and synbiotic up-regulated the colonic expression of T-bet in acute colitis mice, which was associated with the regulation of innate immune responses in colon. The expression of pro-inflammatory cytokines (such as TNF- $\alpha$ and IL-6) was decreased in FOS or synbiotic treated colitis mice, while the expression of anti-inflammatory cytokines (such as IL-10) was increased. In summary, the modulation of gut microbiota through FOS offers a bright prospect for the intervention of inflammatory bowel diseases, especially in the acute phase of disease. The application of metabolomics analysis and the determination of specific immune cell subpopulation at colonic mucosa might be helpful to further explore their action mechanisms in follow-up studies.

\section{Author contributions}

Minjing Liao contributed to the conceptualization, funding acquisition and writing. Yuanfang Zhang, Yilan Qiu, Zhengchun $\mathrm{Wu}$ and Xiaoqi Zeng contributed to the investigation. Zhihong Zhong and Yiliang Zeng contributed to the resources. Li Xiong and $\mathrm{Yu}$ Wen contributed to the funding acquisition and resources. Rushi Liu contributed to the supervision and funding acquisition.

\section{Conflicts of interest}

There are no conflicts to declare.

\section{Acknowledgements}

We thank the support of grants from Scientific Research Fund of Hunan Provincial Education Department (No. 18 B033 and 2019A293), Hunan Provincial Key Research and Development Program (No. 2019SK2042), Hunan Provincial Natural Science Foundation of China (No. 2019JJ10002) and National Natural Science Foundation of China (No. 31072141, 81970569, 81970655, 81773293).

\section{References}

1 S. C. Ng, H. Y. Shi, N. Hamidi, F. E. Underwood, W. Tang, E. I. Benchimol, et al., Worldwide incidence and prevalence of inflammatory bowel disease in the 21st century: a systematic review of population-based studies, Lancet, 2018, 390(10114), 2769-2778.

2 J. Zhao, S. C. Ng, Y. Lei, F. Yi, J. Li, L. Yu, et al., First prospective, population-based inflammatory bowel disease incidence study in mainland of China: the emergence of "western" disease, Inflammatory Bowel Dis., 2013, 19(9), 1839-1845.

3 K. O. Chudy-Onwugaje, K. E. Christian, F. A. Farraye and R. K. Cross, A State-of-the-Art Review of New and Emerging Therapies for the Treatment of IBD, Inflammatory Bowel Dis., 2019, 25(5), 820-830.

4 A. Kaser, S. Zeissig and R. S. Blumberg, Inflammatory bowel disease, Annu. Rev. Immunol., 2010, 28, 573-621. 
5 J. Halfvarson, C. J. Brislawn, R. Lamendella, Y. VazquezBaeza, W. A. Walters, L. M. Bramer, et al., Dynamics of the human gut microbiome in inflammatory bowel disease, Nat. Microbiol., 2017, 2, 17004.

6 A. D. Kostic, R. J. Xavier and D. Gevers, The microbiome in inflammatory bowel disease: current status and the future ahead, Gastroenterology, 2014, 146(6), 1489-1499.

7 R. B. Sartor, Therapeutic manipulation of the enteric microflora in inflammatory bowel diseases: antibiotics, probiotics, and prebiotics, Gastroenterology, 2004, 126(6), 1620-1633.

8 T. Zuo and S. C. Ng, The Gut Microbiota in the Pathogenesis and Therapeutics of Inflammatory Bowel Disease, Front. Microbiol., 2018, 9, 2247.

9 M. J. Saez-Lara, C. Gomez-Llorente, J. Plaza-Diaz and A. Gil, The role of probiotic lactic acid bacteria and bifidobacteria in the prevention and treatment of inflammatory bowel disease and other related diseases: a systematic review of randomized human clinical trials, BioMed Res. Int., 2015, 2015, 505878.

10 D. Jakubczyk, K. Leszczyńska and S. Górska, The Effectiveness of Probiotics in the Treatment of Inflammatory Bowel Disease (IBD)-A Critical Review, Nutrients, 2020, 12(7), 1973.

11 M. S. Geier, R. N. Butler and G. S. Howarth, Inflammatory bowel disease: current insights into pathogenesis and new therapeutic options; probiotics, prebiotics and synbiotics, Int. J. Food Microbiol., 2007, 115(1), 1-11.

12 S. W. Kim, H. M. Kim, K. M. Yang, S. A. Kim, S. K. Kim, M. J. An, et al., Bifidobacterium lactis inhibits NF-kappaB in intestinal epithelial cells and prevents acute colitis and colitis-associated colon cancer in mice, Inflammatory Bowel Dis., 2010, 16(9), 1514-1525.

13 J. Dicksved, O. Schreiber, B. Willing, J. Petersson, S. Rang, M. Phillipson, et al., Lactobacillus reuteri maintains a functional mucosal barrier during DSS treatment despite mucus layer dysfunction, PLoS One, 2012, 7(9), e46399.

14 D. Paveljšek, P. Juvan, R. Košir, D. Rozman, B. Hacin, K. Ivičak-Kocjan, et al., Lactobacillus fermentum L930BB and Bifidobacterium animalis subsp. animalis IM386 initiate signalling pathways involved in intestinal epithelial barrier protection, Benefic. Microbes, 2018, 9(3), 515-525.

15 C. Wong, P.J. Harris and L.R. Ferguson, Potential Benefits of Dietary Fibre Intervention in Inflammatory Bowel Disease, Int. J. Mol. Sci., 2016, 17(6), 919.

16 M. P. L. Guarino, A. Altomare, S. Emerenziani, C. Di Rosa, M. Ribolsi, P. Balestrieri, et al., Mechanisms of Action of Prebiotics and Their Effects on Gastro-Intestinal Disorders in Adults, Nutrients, 2020, 12(4), 1037.

17 J. Winkler, R. Butler and E. Symonds, Fructo-oligosaccharide reduces inflammation in a dextran sodium sulphate mouse model of colitis, Dig. Dis. Sci., 2007, 52(1), 52-58.

18 M. E. Rodríguez-Cabezas, D. Camuesco, B. Arribas, N. Garrido-Mesa, M. Comalada, E. Bailón, et al., The combination of fructooligosaccharides and resistant starch shows prebiotic additive effects in rats, Clin. Nutr., 2010, 29(6), 832-839.

19 F. Capitán-Cañadas, B. Ocón, C. J. Aranda, A. Anzola, M. D. Suárez, A. Zarzuelo, et al., Fructooligosaccharides exert intestinal anti-inflammatory activity in the CD4+CD62L+T cell transfer model of colitis in C57BL/6J mice, Eur. J. Nutr., 2016, 55(4), 1445-1454.

20 J. O. Lindsay, K. Whelan, A. J. Stagg, P. Gobin, H. O. AlHassi, N. Rayment, et al., Clinical, microbiological, and immunological effects of fructo-oligosaccharide in patients with Crohn's disease, Gut, 2006, 55(3), 348-355.

21 J. L. Benjamin, C. R. Hedin, A. Koutsoumpas, S. C. Ng, N. E. McCarthy, A. L. Hart, et al., Randomised, doubleblind, placebo-controlled trial of fructo-oligosaccharides in active Crohn's disease, Gut, 2011, 60(7), 923-929.

22 S. J. Son, J. H. Koh, M. R. Park, S. Ryu, W. J. Lee, B. Yun, et al., Effect of the Lactobacillus rhamnosus strain GG and tagatose as a synbiotic combination in a dextran sulfate sodium-induced colitis murine model, J. Dairy Sci., 2019, 102(4), 2844-2853.

23 K. Sheng, S. He, M. Sun, G. Zhang, X. Kong, J. Wang, et al., Synbiotic supplementation containing Bifidobacterium infantis and xylooligosaccharides alleviates dextran sulfate sodium-induced ulcerative colitis, Food Funct., 2020, 11(5), 3964-3974.

24 Z. Liu, F. Liu, W. Wang, C. Sun, D. Gao, J. Ma, et al., Study of the alleviation effects of a combination of Lactobacillus rhamnosus and inulin on mice with colitis, Food Funct., 2020, 11(5), 3823-3837.

25 S. Wirtz, V. Popp, M. Kindermann, K. Gerlach, B. Weigmann, S. Fichtner-Feigl, et al., Chemically induced mouse models of acute and chronic intestinal inflammation, Nat. Protoc., 2017, 12(7), 1295-1309.

26 A. L. M. Silveira, A. V. M. Ferreira, M. C. de Oliveira, M. A. Rachid, L. F. da Cunha Sousa, F. Dos Santos Martins, et al., Preventive rather than therapeutic treatment with high fiber diet attenuates clinical and inflammatory markers of acute and chronic DSS-induced colitis in mice, Eur. J. Nutr., 2017, 56(1), 179-191.

27 Y. Zhang, P. Wang, C. Xia, Z. Wu, Z. Zhong, Y. Xu, et al., Fructooligosaccharides supplementation mitigated chronic stress-induced intestinal barrier impairment and neuroinflammation in mice, J. Funct. Foods, 2020, 72, 104060.

28 B. Zheng, J. van Bergenhenegouwen, H. J. van de Kant, G. Folkerts, J. Garssen, A. P. Vos, et al., Specific probiotic dietary supplementation leads to different effects during remission and relapse in murine chronic colitis, Benefic. Microbes, 2016, 7(2), 205-213.

29 W. Kruis, P. Fric, J. Pokrotnieks, M. Lukás, B. Fixa, M. Kascák, et al., Maintaining remission of ulcerative colitis with the probiotic Escherichia coli Nissle 1917 is as effective as with standard mesalazine, Gut, 2004, 53(11), 1617-1623.

30 T. M. Chapman, G. L. Plosker and D. P. Figgitt, VSL\#3 probiotic mixture: a review of its use in chronic inflammatory bowel diseases, Drugs, 2006, 66(10), 1371-1387. 
31 J. Plaza-Díaz, F. J. Ruiz-Ojeda, L. M. Vilchez-Padial and A. Gil, Evidence of the Anti-Inflammatory Effects of Probiotics and Synbiotics in Intestinal Chronic Diseases, Nutrients, 2017, 9(6), 555.

32 M. Laffin, T. Perry, H. Park, N. Hotte, R. N. Fedorak, A. Thiesen, et al., Prebiotic Supplementation Following Ileocecal Resection in a Murine Model is Associated With a Loss of Microbial Diversity and Increased Inflammation, Inflammatory Bowel Dis., 2017, 24(1), 101-110.

33 A. Loy, C. Pfann, M. Steinberger, B. Hanson, S. Herp, S. Brugiroux, et al., Lifestyle and Horizontal Gene TransferMediated Evolution of Mucispirillum schaedleri, a Core Member of the Murine Gut Microbiota, mSystems, 2017, 2(1), e00171-16.

34 D. Berry, C. Schwab, G. Milinovich, J. Reichert, K. Ben Mahfoudh, T. Decker, et al., Phylotype-level 16S rRNA analysis reveals new bacterial indicators of health state in acute murine colitis, ISME J., 2012, 6(11), 2091-2106.

35 Z. Xu, W. Chen, Q. Deng, Q. Huang, X. Wang, C. Yang, et al., Flaxseed oligosaccharides alleviate DSS-induced colitis through modulation of gut microbiota and repair of the intestinal barrier in mice, Food Funct., 2020, 11(9), 8077-8088.

36 J. Pujo, C. Petitfils, P. Le Faouder, V. Eeckhaut, G. Payros, S. Maurel, et al. Bacteria-derived long chain fatty acid exhibits anti-inflammatory properties in colitis, Gut, 2020, 70(6), 1088-1097.

37 F. Liu, J. Liu, T. T. Y. Wang, Z. Liu, C. Xue, X. Mao, et al., Molecular and Microbial Signatures Predictive of Prebiotic Action of Neoagarotetraose in a Dextran Sulfate SodiumInduced Murine Colitis Model, Microorganisms, 2020, 8(7), 995.

38 R. Valcheva, P. Koleva, I. Martínez, J. Walter, M. G. Gänzle and L. A. Dieleman, Inulin-type fructans improve active ulcerative colitis associated with microbiota changes and increased short-chain fatty acids levels, Gut Microbes, 2019, 10(3), 334-357.
39 C. Chelakkot, J. Ghim and S. H. Ryu, Mechanisms regulating intestinal barrier integrity and its pathological implications, Exp. Mol. Med., 2018, 50(8), 1-9.

40 S. van der Post, K. S. Jabbar, G. Birchenough, L. Arike, N. Akhtar, H. Sjovall, et al., Structural weakening of the colonic mucus barrier is an early event in ulcerative colitis pathogenesis, Gut, 2019, 68(12), 2142-2151.

41 K. Hiippala, H. Jouhten, A. Ronkainen, A. Hartikainen, V. Kainulainen, J. Jalanka, et al., The Potential of Gut Commensals in Reinforcing Intestinal Barrier Function and Alleviating Inflammation, Nutrients, 2018, 10(8), 988.

42 H. Wang, P. Shi, L. Zuo, J. Dong, J. Zhao, Q. Liu, et al., Dietary Non-digestible Polysaccharides Ameliorate Intestinal Epithelial Barrier Dysfunction in IL-10 Knockout Mice, Journal Crohn's Colitis, 2016, 10(9), 1076-1086.

43 W. T. Uniken Venema, M. D. Voskuil, G. Dijkstra, R. K. Weersma and E. A. Festen, The genetic background of inflammatory bowel disease: from correlation to causality, J. Pathol., 2017, 241(2), 146-158.

44 M. E. Johansson, J. K. Gustafsson, J. Holmén-Larsson, K. S. Jabbar, L. Xia, H. Xu, et al., Bacteria penetrate the normally impenetrable inner colon mucus layer in both murine colitis models and patients with ulcerative colitis, Gut, 2014, 63(2), 281-291.

45 W. S. Garrett, G. M. Lord, S. Punit, G. Lugo-Villarino, S. K. Mazmanian, S. Ito, et al., Communicable ulcerative colitis induced by T-bet deficiency in the innate immune system, Cell, 2007, 131(1), 33-45.

46 R. Mohamed and G. M. Lord, T-bet as a key regulator of mucosal immunity, Immunology, 2016, 147(4), 367-376.

47 N. Garrido-Mesa, J. H. Schroeder, E. Stolarczyk, A. L. Gallagher, J. W. Lo, C. Bailey, et al., T-bet controls intestinal mucosa immune responses via repression of type 2 innate lymphoid cell function, Mucosal Immunol., 2019, 12(1), 51-63. 